\title{
Research on the Design Criterion of Packers and Slips in Pipeline Isolation Tool
}

\author{
Kang Zhang, Shimin Zhang, Qingxin Ding, Pu Gong and Xiaoxiao Zhu \\ China University of Petroleum-Beijing, No.18 Fuxue Road, Changping District Beijing, China
}

\begin{abstract}
With the rapid development of pipeline industry, the pipeline maintenance technology using isolation tool has got developed widely in the global scope. The packers and slips are the determining parts for isolation reliability and their design criterions are essential to investigate. This paper firstly studied the contact pressure between the packers and pipeline through theoretical and simulation methods. And then the modified model has been achieved according to the analysis results. Finally, the design criterions of packers and slips have been presented accordingly. The results of this research has great meaning and provide efficiency and convenience for tool designing in engineering.
\end{abstract}

\section{Introduction}

Transmission pipeline has become the main way to deliver fluids such as oil and gas due to its advantages of safety, efficiency and economy. In order to reduce the economic loss caused by the accidents like corrosions and cracks, the pipeline maintenance technology using isolation tool [1-3] has been widely developed in recent years.

The pipeline isolation tool is propelled from pig launcher via a flow of fluid and stops in the desired position inside pipeline to anchor and isolate $[4,5]$. The packers and slips are the most important parts which determine the reliability of pipeline isolation. However, research on the design of packers and slips is scarcely found in the literatures, most of the available knowledge is mainly based on the experiences of designer, which made it inefficient and error prone. As a result, the research on design criterion of packers and slips is necessary.

In this paper, the analysis methods and design criterion of packers and slips have been proposed, and the results of this research will be helpful for providing some academic basis for engineering applications.

\section{Analysis on contact pressure between packers and pipeline}

The isolation process is shown in Fig. 1, the packers and slips expand under the axial compression load $F_{Z}$ during the isolation process, the contact pressure between packers and pipeline is $P_{k}$. It is assumed that the differential pressure which the tool could isolate is equal to the contact pressure. So the contact pressure is the essential parameter and needs to be studied in advance.

\subsection{Theoretical analysis}

The simplified force model of packer is shown in Fig. 2. The packer will deform under the axial compression load $F_{Z}$, and the axial stress is:

$$
\sigma_{z}=\frac{F_{Z}}{S}
$$

Where, $S$ is the section area of packer.

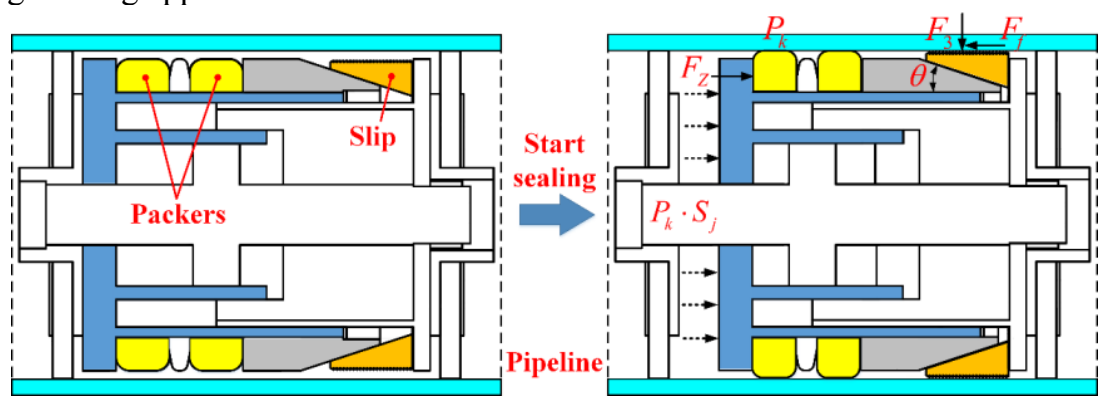

Figure 1. The schematic diagram of isolation process. 


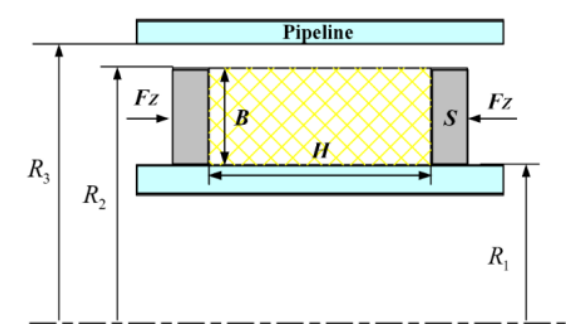

Figure 2. The simplified force model of packer.

Under the axial compression load, the deformation of packer could be divided into two phases: in phase 1, the packer will deform freely until contacting with pipeline; in phase 2, after contacting with pipeline, the contact pressure will increase with the rise of axial compression load. So the axial stress could be written as:

$$
\sigma_{z}=\sigma_{z}{ }^{1}+\sigma_{z}^{2}
$$

Where, $\sigma_{z}{ }^{1}$ is the axial stress of phase 1 , and $\sigma_{z}^{1}=G\left[\frac{R_{3}^{2}-R_{1}^{2}}{R_{2}^{2}-R_{1}^{2}}-\left(\frac{R_{2}^{2}-R_{1}^{2}}{R_{3}^{2}-R_{1}^{2}}\right)^{2}\right], G$ is the modulus of rigidity. $\sigma_{z}^{2}$ is the axial stress of phase 2 and it could be rewritten as:

$$
\sigma_{z}^{2}=\sigma_{z}-\sigma_{z}{ }^{1}=\frac{F_{Z}}{S}-G\left[\frac{R_{3}^{2}-R_{1}^{2}}{R_{2}^{2}-R_{1}^{2}}-\left(\frac{R_{2}^{2}-R_{1}^{2}}{R_{3}^{2}-R_{1}^{2}}\right)^{2}\right] \text { (3) }
$$

According to generalized Hooke law, the radial stress $\sigma_{r}$ and axial stress $\sigma_{z}^{2}$ could be written as:

$$
\begin{gathered}
\sigma_{r}=\frac{E}{(1-2 \mu)(1+\mu)}\left[(1-\mu) \varepsilon_{r}+\mu\left(\varepsilon_{\theta}+\varepsilon_{z}\right)\right] \\
\sigma_{z}^{2}=\frac{E}{(1-2 \mu)(1+\mu)}\left[(1-\mu) \varepsilon_{z}+\mu\left(\varepsilon_{r}+\varepsilon_{\theta}\right)\right]
\end{gathered}
$$

Where, $\mu$ is the Poisson's ratio. $\varepsilon_{r}$ and $\varepsilon_{\theta}$ are the strain of radial and circumference, respectively. And $\varepsilon_{r}=\varepsilon_{\theta}=0$. So the radial strain could be written as:

$$
\sigma_{r}=\frac{\mu}{1-\mu} \sigma_{z}^{2}
$$

Therefore, the contact pressure between the packer and pipeline is:

$$
P_{k}=\sigma_{r}=\frac{\mu}{1-\mu}\left[\frac{F_{Z}}{S}-G \frac{\left(R_{3}^{2}-R_{1}^{2}\right)^{3}-\left(R_{2}^{2}-R_{1}^{2}\right)^{3}}{\left(R_{2}^{2}-R_{1}^{2}\right)\left(R_{3}^{2}-R_{1}^{2}\right)^{2}}\right]
$$

In order to improve the accuracy, the friction of packer has been taken into consideration, and the contact pressure [6] could be rewritten as Eq. (8):

$$
P_{k}=\left\{\begin{array}{lr}
0 & \left(0 \leq F_{Z}<\frac{2 \pi E R_{3}\left(R_{3}-R_{2}\right)}{1+\mu}\right) \\
\frac{\mu}{1-\mu}\left[\frac{F_{Z}}{\pi\left(R_{3}^{2}-R_{1}^{2}\right)}-\frac{2 E R_{3}}{(1+\mu)} \cdot\left(\frac{R_{3}-R_{2}}{\left.R_{3}^{2}-R_{1}^{2}\right)}\right]\right. & \left(F_{Z} \geq \frac{2 \pi E R_{3}\left(R_{3}-R_{2}\right)}{1+\mu}\right)
\end{array}\right.
$$

\subsection{Error correction of theoretical model}

In order to verify the theoretical model, the contact pressure between packer and pipeline was also analysed through simulation method. In the simulation, the Mooney-Rivlin model was used in rubber material properties. The strain energy density function is: $W=C_{10}\left(I_{1}-3\right)+C_{01}\left(I_{2}-3\right), W$ is strain potential, $I_{1}$ and $I_{2}$ are deformation tensor, $C_{10}$ and $C_{01}$ are parameters of Mooney-Rivlin Model. The other parameters selected in this simulation is shown in Table 1.

Table 1. The parameters selected in the simulation.

\begin{tabular}{|c|c|c|c|c|c|c|}
\hline $\begin{array}{c}\mathrm{D} \\
(\mathrm{mm})\end{array}$ & $\begin{array}{c}R_{1} \\
(\mathrm{~mm})\end{array}$ & $\begin{array}{c}R_{2} \\
(\mathrm{~mm})\end{array}$ & $\begin{array}{c}R_{3} \\
(\mathrm{~mm})\end{array}$ & $\begin{array}{c}H \\
(\mathrm{~mm})\end{array}$ & $\begin{array}{c}B \\
(\mathrm{~mm})\end{array}$ & $\begin{array}{c}\text { IRHD } \\
(\mathrm{HS})\end{array}$ \\
\hline 508 & 182 & 232 & 244 & 80 & 50 & 70 \\
\hline
\end{tabular}

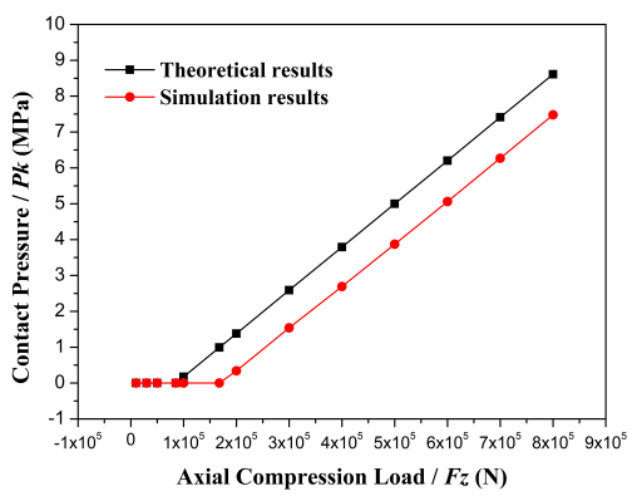

Figure 3. The comparison of theoretical and simulation results.

Fig. 3 shows the comparison of theoretical and simulation results. It is obvious from Fig. 3 that there appear to be linear relationship between the contact pressure and axial compression load. Also, the slopes of these two lines were almost equal, while the error was mainly due to the intercept. The reason resulting in the error is there will be frictional forces existing between moving segments and they are hard to be calculated precisely. Therefore, a correction factor $\lambda$ has been introduced to the Eq. 9:

$$
P_{k}=\left[\frac{\mu}{1-\mu} \cdot \frac{1}{\pi\left(R_{3}^{2}-R_{1}^{2}\right)}\right] \cdot F_{Z}-\lambda \cdot\left[\frac{\mu}{1-\mu} \cdot \frac{2 E R_{3}}{(1+\mu)} \cdot\left(\frac{R_{3}-R_{2}}{R_{3}^{2}-R_{1}^{2}}\right)\right] \text { (9) }
$$

Through comparing the results of theory and simulation, the correction factor could be determined as $\lambda=1.898$. So the modified equation could be written as:

$$
P_{k}=\left\{\begin{array}{rr}
0 & \left(0 \leq F_{Z}<1.898 \times \frac{2 \pi E R_{3}\left(R_{3}-R_{2}\right)}{1+\mu}\right) \\
{\left[\frac{\mu}{1-\mu} \cdot \frac{1}{\pi\left(R_{3}^{2}-R_{1}^{2}\right)}\right] \cdot F_{Z}-1.898\left[\frac{\mu}{1-\mu} \cdot \frac{2 E R_{3}}{(1+\mu)} \cdot\left(\frac{R_{3}-R_{2}}{\left.R_{3}^{2}-R_{1}^{2}\right)}\right) \quad\left(F_{Z} \geq 1.898 \times \frac{2 \pi E R_{3}\left(R_{3}-R_{2}\right)}{1+\mu}\right)\right.}
\end{array}\right.
$$




\section{Design criterion of packers and slips}

\subsection{Design criterion of packers}

The structural optimization of packers and slips has been widely developed [7-11]. While another important design criterion of packer is self-sealing. As shown in Fig. 4, when the axial compression load is $F_{Z}$, the contact pressure between packer and pipeline is $P_{k}$, so the axial load generated by differential pressure is $F_{2}=P_{k} \cdot S_{j}$, where $S_{j}$ is the sectional area of isolation tool. With the increase of $F_{Z}$ and $P_{k}$, if $F_{2}>F_{Z}$, which means that the axial compression load needed for isolation could be provided totally by differential pressure, the self-sealing achieved. Therefore, the design criterion of packer is:

$$
\left\{\begin{array}{l}
F_{2}=P_{k} \cdot S_{j} \\
F_{2}>F_{Z}
\end{array}\right.
$$

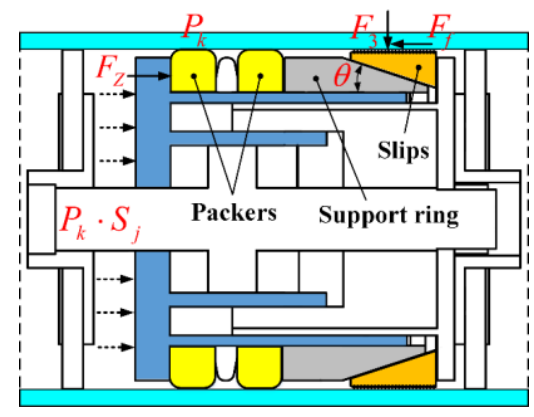

Figure 4. The schematic diagram of force analysis.

In order to make it more clear to understand easily, an example has been analyzed in this paper. The parameters selected in this analysis are shown in Table 1, and the results are shown in Fig. 5.

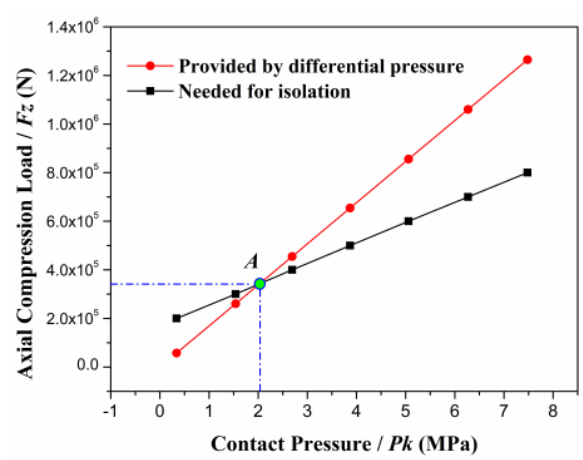

Figure 5. The analysis of self-sealing of packer.
It is clear from Fig. 5 that below the intersection point $A(2.02 \mathrm{MPa}, 340 \mathrm{kN})$, the axial compression load needed for isolation was smaller than that provided by differential pressure. However, with the rise of contact pressure, the gap between them narrowed gradually and when it reached beyond point $A$, the axial load provided by differential pressure was consistently bigger than that needed for isolation. It can be concluded that the axial compression load needed for isolation is only $340 \mathrm{kN}$, generating the contact pressure $2.02 \mathrm{MPa}$, the packer could achieve self-sealing.

\subsection{Design criterion of slips}

The force diagram of slips is also shown in Fig. 4. According to force balance, the normal force of slip is $F_{3}=F_{Z} / \tan \theta$, and the frictional force of the slip is $F_{f}=\mu_{f} \cdot F_{3}=\mu_{f} \cdot F_{Z} / \tan \theta$, where $\mu_{f}$ is the frictional coefficient, $\theta$ is the angle of support ring. The main function of slips is to anchor the tool in pipe, so the design criterion of slips including:

(1) In order to ensure the reliability of anchor, the frictional force needs to be larger than axial load provided by differential pressure.

$$
F_{f}>F_{2}
$$

(2) In order to achieve self-anchoring, the axial load provided by differential pressure needs to be larger than axial compression load needed for anchoring.

$$
F_{2}>F_{Z}
$$

(3) The maximum contact stress needs to be smaller than the allowable stress of slip and pipeline.

$$
\frac{F_{3 \max }}{S_{n}}<80 \% \cdot \min \left\{\sigma_{S 1}, \sigma_{S 2}\right\}
$$

Where, $S_{n}$ is radial sectional area of slips, $\sigma_{S 1}$ is the allowable stress of slip and $\sigma_{S 2}$ is the allowable stress of pipeline.

(4) The contact pressure of packer needs to be larger than design requirements under maximum axial compression load.

$$
P_{k \max } \geq P_{D}
$$

(5) The angle of support ring needs to be larger than self-lock angle.

$$
\theta>\text { self-lock angle }\left(18^{\circ}\right)
$$

Above all, the design criterion of slips can be written as Eq. (17):

$$
\left\{\begin{array}{l}
\frac{3.796 \pi E \cdot R_{3}\left(R_{3}-R_{2}\right) S_{j} \cdot \mu}{(1+\mu)\left[S_{j} \cdot \mu-\pi(1-\mu)\left(R_{3}^{2}-R_{1}^{2}\right)\right]}<F_{Z}<\frac{3.796 \pi E \cdot R_{3}\left(R_{3}-R_{2}\right) S_{j} \cdot \mu \cdot \tan \theta}{(1+\mu)\left[S_{j} \cdot \mu \cdot \tan \theta-\mu_{f} \cdot \pi(1-\mu)\left(R_{3}^{2}-R_{1}^{2}\right)\right]} \\
\left(\frac{1-\mu}{\mu} P_{k \max }+\frac{3.796 E \cdot R_{3}\left(R_{3}-R_{2}\right)}{(1+\mu)\left(R_{3}^{2}-R_{1}^{2}\right)}\right) \frac{\pi\left(R_{3}^{2}-R_{1}^{2}\right)}{\tan \theta \cdot S_{n}}<80 \% \cdot \min \left\{\sigma_{S 1}, \sigma_{S 2}\right\} \\
P_{k \max }=\left[\frac{\mu}{1-\mu} \cdot \frac{1}{\pi\left(R_{3}^{2}-R_{1}^{2}\right)}\right] \cdot F_{Z \max }-1.898\left[\frac{\mu}{1-\mu} \cdot \frac{2 E R_{3}}{(1+\mu)} \cdot\left(\frac{R_{3}-R_{2}}{\left.R_{3}^{2}-R_{1}^{2}\right)}\right] \geq P_{D}\right. \\
\theta>\text { self-lock angle }\left(18^{\circ}\right)
\end{array}\right.
$$




\section{Conclusion}

This paper investigated the design criterion of packers and slips. The conclusions can be drawn as follows:

1). The contact pressure between packers and pipeline has been analyzed through theoretical and simulation methods. And the error correction model has been achieved by comparing the results to improve its precision.

2). The design criterions of packers and slips have been presented in this paper, it is essential for the design of packers and slips, and make it more efficient and convenient.

The results of this research provided not only theoretical basis for design criterion, but also guidance for pipeline isolation tool in engineering application.

\section{Acknowledgements}

This work was supported by the National Natural Science Foundation of China (No. 51509259).

\section{References}

1. E. Tveit, J. Aleksandersen, SPE Asia Pacific Oil and Gas Conference and Exhibition (2000)
2. B.J. Zhao, C.R. Li, J.H. Zhang, International Offshore and Polar Engineering Conference (2010)

3. J. Aleksandersen, H. Syse, U.S. Patent 7,878,221. (2011)

4. J. Aleksandersen, E. Tveit, International Offshore and Polar Engineering Conference (2001)

5. M.R.G Lie, M.N. Muangsuankwan, ASME India International Oil and Gas Pipeline Conference (2015)

6. S.K. Tong, Oil Field Equipment. 41, 7 (2012) (In Chinese)

7. Z.C. Lin, Applied Mechanics \& Materials. 423, 4 (2013)

8. J. Wang, X. Han, J. Wang, Asia-Pacific Energy Equipment Engineering Research Conference (2015)

9. D.X. Li, H.L. Zhao, S.M. Zhang, Adv. Mater. Res. 199, 6 (2011)

10. M. Ma, W. Jia, Y. Bu, Open Access Library Journal. 01, 8 (2014)

11. Z.P. Guo, W. Guo, Y.F. Wang, Applied Mechanics \& Materials. 252, 3 (2013) 